\title{
Improved Genetic Algorithm (VNS-GA) using polar coordinate classification for workload balanced multiple Traveling Salesman Problem (mTSP)
}

\author{
Wang, Y.D. ${ }^{a}$, Lu, X.C. ${ }^{b,}{ }^{*}$, Shen, J.R. ${ }^{c}$ \\ ${ }^{a}$ Beijing Jiaotong University, Shangyuan Village, Haidian District, Beijing, P.R. China \\ ${ }^{b}$ Beijing Jiaotong University, Shangyuan Village, Haidian District, Beijing, P.R. China \\ 'Beijing Capital Agribusiness \& Food Group Co., Ltd., Xicheng District, Beijing, P.R. China
}

\begin{abstract}
A B S TRACT
The multiple traveling salesman problem (mTSP) is an extension of the traveling salesman problem (TSP), which has wider applications in real life than the traveling salesman problem such as transportation and delivery, task allocation, etc. In this paper, an improved genetic algorithm (VNS-GA) that uses polar coordinate classification to generate the initial solutions is proposed. It integrates the variable neighbourhood algorithm to solve the multiple objective optimization of the mTSP with workload balance. Aiming to workload balance, the first design of this paper is about generating initial solutions based on the polar coordinate classification. Then a distance comparison insertion operator is designed as a neighbourhood action for allocating paths in a targeted manner. Finally, the neighbourhood descent process in the variable neighbourhood algorithm is fused into the genetic algorithm for the expansion of search space. The improved algorithm is tested on the TSPLIB standard data set and compared with other genetic algorithms. The results show that the improved genetic algorithm can increase computational efficiency and obtain a better solution for workload balance and this algorithm has wild applications in real life such as multiple robots task allocation, school bus routing problem and other optimization problems.
\end{abstract}

\section{ARTICLE INFO}

Keywords:

Multiple traveling salesman

problem (mTSP);

Workload balance;

Variable neighbourhood search

algorithm (VNS);

Genetic algorithm (GA);

Polar coordinates;

Classification

*Corresponding author:

xclu@bjtu.edu.cn

(Lu, X.C.)

Article history:

Received 4 June 2021

Revised 15 June 2021

Accepted 17 June 2021

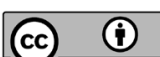

Content from this work may be used under the terms of the Creative Commons Attribution 4.0 International the Creative Commons Attribution 4.0 International Licence (CC BY 4.0). Any further distribution of this work must maintain attribution to the author(s) and the title of
the work, journal citation and DOI.

\section{References}

[1] Bektas, T. (2006). The multiple traveling salesman problem: An overview of formulations and solution procedures, Omega, Vol. 34, No. 3, 209-219, doi: 10.1016/i.omega.2004.10.004.

[2] Bostanci, B., Karaağaç, A. (2019). Investigating the shortest survey route in a GNSS traverse network, Tehnički Vjesnik - Technical Gazette, Vol. 26, No. 2, 355-362, doi: 10.17559/TV-20170924174221.

[3] Iqbal Ali, A., Kennington, J.L. (1986). The asymmetric M-traveling salesmen problem: A duality based branchand-bound algorithm, Discrete Applied Mathematics, Vol. 13, No. 2-3, 259-276, doi: 10.1016/0166-218X(86) 90087-9.

[4] Gavish, B., Srikanth, K. (1986). An optimal solution method for large-scale multiple traveling salesman problems, Operations Research, Vol. 34, No. 5, 698-717, doi: 10.1287/opre.34.5.698.

[5] Yu, Q.S., Lin, D.M., Wang, D. (2012). An overview of multiple traveling salesman problem, Value Engineering, Vol. 31, No. 2, 166-168, doi: 10.14018/i.cnki.cn13-1085/n.2012.02.143.

[6] Carter, A.E., Ragsdale, C.T. (2006). A new approach to solving the multiple traveling salesperson problem using genetic algorithms, European Journal of Operational Research, Vol. 175, No. 1, 246-257, doi: 10.1016/i.ejor.2005. 
$\underline{04.027}$.

[7] Singh, A., Baghel, A.S. (2009). A new grouping genetic algorithm approach to the multiple traveling salesperson problem, Soft Computing, Vol. 13, 95-101, doi: 10.1007/s00500-008-0312-1.

[8] Zhou, W., Li, Y. (2010). An improved genetic algorithm for multiple traveling salesman problem, In: Proceedings of $2^{\text {nd }}$ International Asia Conference on Informatics in Control, Automation and Robotics (CAR 2010), Wuhan, China, 493-495, doi: 10.1109/CAR.2010.5456787.

[9] Koh, S.P., bin Aris, I., Ho, C.K., Bashi, S.M. (2006). Design and performance optimization of a multi-TSP (Traveling Salesman Problem) algorithm, Artificial Intelligence and Machine Learning AIML, Vol. 6, No. 3, 29-33.

[10] Hu, S.J., Lu, H.Y., Huang, Y., Xu, K.B. (2019). Improved genetic algorithm for solving multiple traveling salesman problem with balanced workload, Computer Engineering and Applications, Vol. 55, No. 17, 150-155.

[11] Guo, S. (2019). Solutions space analysis of MTSP and application in VRP optimization, Beijing University of Posts and Telecommunications, Beijing, China, from https://kns.cnki.net/KCMS/detail/detail.aspx?dbname=CMFD201902\&filename=1019113259.nh, accessed April $11,2021$.

[12] Lu, Z., Zhang, K., He, J., Niu, Y. (2016), Applying K-means clustering and genetic algorithm for Solving MTSP, In: Gong, M., Pan, L., Song, T., Zhang, G. (eds.), Bio-inspired Computing - Theories and Applications, Springer Singapore, 278-284, doi: 10.1007/978-981-10-3614-9 34.

[13] Cheikhrouhou, O., Khoufi, I. (2021). A comprehensive survey on the multiple travelling salesman problem: Applications, approaches and taxonom, Computer Science Review, Vol. 40, doi: /10.1016/j.cosrev.2021.100369.

[14] Pan, J., Wang, D. (2006). An ant colony optimization algorithm for multiple travelling salesman problem, In: Proceedings of the First International Conference on Innovative Computing, Information and Control - Volume I (ICICIC'06), Beijing, China, 210-213, doi: 10.1109/icicic.2006.40.

[15] Venkatesh, P., Singh, A. (2015). Two metaheuristic approaches for the multiple traveling salesperson problem, Applied Soft Computing, Vol. 26, 74-89, doi: 10.1016/j.asoc.2014.09.029.

[16] Ryan, J.L., Bailey, T.G., Moore, J.T., Carlton, W.B. (1998), Reactive tabu search in unmanned aerial reconnaissance simulations, In: Proceedings of the 30th 1998 Winter Simulation Conference. Proceedings (Cat. No.98CH36274), Washington, USA, Vol. 1, 873-879, doi: 10.1109/wsc.1998.745084.

[17] Song, C.H., Lee, K., Lee, W.D. (2003). Extended simulated annealing for augmented TSP and multi-salesmen TSP, In: Proceedings of the International Joint Conference on Neural Networks 2003, Oregon, USA, Vol. 3, 2340-2343, doi: 10.1109/IJCNN.2003.1223777.

[18] $\mathrm{Hu}$, Y., Yao, Y., Lee, W.S. (2020). A reinforcement learning approach for optimizing multiple traveling salesman problems over graphs, Knowledge-Based Systems, Vol. 204, Article No. 106244, doi: 10.1016/j.knosys.2020. 106244.

[19] Bonz, J. (2021). Application of a multi-objective multi traveling salesperson problem with time windows, Public Transport, Vol. 13, 35-57, doi: 10.1007/s12469-020-00258-6.

[20] Liu, H., Zhang, H., Xu, Y. (2021). The m-Steiner traveling salesman problem with online edge blockages, Journal of Combinatorial Optimization, Vol. 41, 844-860, doi: 10.1007/s10878-021-00720-6.

[21] Dong, H.Y., Huang, M., Wang, X.W., Zheng, B.L. (2009). Review of variable neighborhood search algorithm, Control Engineering of China, Vol. 16, No. 2, 1-5.

[22] Li, J., Guo, Y.H. (2001). Theory and method of optimal scheduling of logistics distribution vehicles, China Fortune Press, Beijing, China.

[23] Min, J.N., Jin, C., Lu, L.J. (2019). Split-delivery vehicle routing problems based on a multi-restart improved sweep approach, International Journal of Simulation Modelling, Vol. 18, No. 4, 708-719, doi: 10.2507/IJSIMM18(4)C019.

[24] Min, J.N., Jin, C., Lu, L.J. (2019). Maximum-minimum distance clustering method for split-delivery vehicle-routing problem: Case studies and performance comparisons, Advances in Production Engineering \& Management, Vol. 14, No. 1, 125-135, doi: 10.14743/apem2019.1.316.

[25] Xiong, C., Wu, H.P., Li, B. (2010). Improved genetic algorithm for solving MTSP, In: Proceedings of the $4^{\text {th }}$ China Intelligent Computing Conference, Beijing, China, 143-149.

[26] $\mathrm{Hu}, \mathrm{S} . J$. (2019). Research on multiple traveling salesman problem based on improved genetic algorithm, Master Thesis, Jiangnan University, Jiangnan, China. 


\section{APEM}

\title{
Izboljšan genetski algoritem (VNS-GA) z uporabo klasifikacije $s$ pomočjo polarnih koordinat za problem več trgovskih potnikov (mTSP) z uravnoteženo delovno obremenitvijo
}

\author{
Wang, Y.D. ${ }^{a}$, Lu, X.C. ${ }^{b,}$, , Shen, J.R. ${ }^{c}$ \\ aBeijing Jiaotong University, Shangyuan Village, Haidian District, Beijing, P.R. China \\ bBeijing Jiaotong University, Shangyuan Village, Haidian District, Beijing, P.R. China \\ 'Beijing Capital Agribusiness \& Food Group Co., Ltd., Xicheng District, Beijing, P.R. China
}

\section{POVZETEK}

Problem več trgovskih potnikov (mTSP) je razširitev problema trgovskega potnika (TSP) in ima v resničnem življenju širše področje uporabe kot problem trgovskega potnika, npr. prevoz in dostava, dodeljevanje nalog itd. V članku je predlagan izboljšan genetski algoritem (VNS-GA), ki uporablja klasifikacijo s pomočjo polarnih koordinat za ustvarjanje začetnih rešitev in vključuje algoritem spremenljive soseske za reševanje večkriterijske optimizacije mTSP z uravnoteženjem delovne obremenitve. Z namenom uravnoteženja delovne obremenitve je najprej predstavljeno ustvarjanje začetnih rešitev na podlagi klasifikacije s pomočjo polarnih koordinat. Za ciljno dodeljevanje poti je nato vključen operator primerjave razdalje. Na koncu se - za razširitev iskalnega prostora - postopek spuščanja po soseščini v algoritmu spremenljive soseske vključi v genetski algoritem. Izboljšani algoritem je testiran na standardnem nizu podatkov TSPLIB in primerjan $\mathrm{z}$ drugimi genetskimi algoritmi. Rezultati kažejo, da lahko izboljšan genetski algoritem poveča računsko učinkovitost in pridobi boljšo rešitev za ravnovesje delovne obremenitve; ta algoritem pa ima mnogo možnosti uporabe v resničnem življenju, kot so dodeljevanje nalog več robotom, usmerjanje poti šolskega avtobusa in drugi optimizacijski problemi.

\section{PODATKI O ČLANKU}

Ključne besede:

Problem več trgovskih potnikov (mTSP);

Ravnovesje delovne obremenitve; Algoritem spremenljive soseske (VNS);

Genetski algoritem (GA);

Polarne koordinate;

Klasifikacija

*Kontaktna oseba: xclu@bjtu.edu.cn

(Lu, X.C.)

Zgodovina članka:

Prejet 4. junija 2021

Popravljen 15. junija 2021

Sprejet 17. junija 2021

\section{(i)}

Content from this work may be used under the terms of the Creative Commons Attribution 4.0 International Licence (CC BY 4.0). Any further distribution of this work must maintain attribution to the author(s) and the title of
the work, journal citation and DOI. 